\title{
THEORÍA
}

NÚMERO 41 | DICIEMBRE 2021 - MAYO 2022 | 123-144

DOI: 10.22201/ffyl.16656415p.2021.41.1565

REVISTA DEL COLEGIO DE FILOSOFÍA

Recibido: 1-07-2021| Aceptado: 8-10-2021

\section{El yo SITUAdO Y RELACiONAL EN Nishida y WATSUji: UNA APROXIMACIÓN DESDE EL PARADIGMA DE LA INTIMIDAD DE KASULIS ${ }^{1}$ \\ The Situated and Relational I in Nishida and Watsuj: An Approach from Kasuli's Intimacy Paradigm}

\author{
Raquel Bouso \\ Universitat Pompeu Fabra | España \\ Contacto: raquel.bouso@upf.edu
}

\section{Resumen}

En su obra Intimacy or Integrity: Philosophy and Cultural Difference (originalmente 1988 Gilbert Ryle Lectures, publicada en 2002), Thomas P. Kasulis identifica dos tipos de orientaciones "intimidad" e "integridad" que condicionan tanto las relaciones interpersonales como nuestra relación con el mundo. Si bien ambas orientaciones se pueden hallar tanto en las tradiciones filosóficas occidentales como en las asiáticas, puede decirse que la primera predomina en buena parte de la filosofía japonesa. A partir del paradigma de la intimidad descrito por Kasulis, examinaremos dos creaciones conceptuales de Nishida Kitarō (1870-1945) y Watsuji Tetsurō (18891960) respectivamente, el "yo como lugar" y el "ambiente geocultural". De este modo, mostraremos cómo emerge un modo de comprender el "yo" como algo situado y relacional que nos permite pensar un modo de ser ecológico-comunitario.

Palabras clave: Filosofía japonesa, Nishida, Watsuji, lugar, ambiente geocultural, basho, fūdo, intimidad

\begin{abstract}
In his work Intimacy or Integrity: Philosophy and Cultural Difference (originally 1988 Gilbert Ryle Lectures, published in 2002), Thomas P. Kasulis identifies two types of orientations "intimacy" and "integrity" that condition both interpersonal relationships and our relationship with the world. Although the two orientations can be found in both Western and Asian philosophical traditions, it can be said that the former predominates in much of Japanese philosophy. Starting from the paradigm of intimacy described by Kasulis, we will examine two conceptual creations by Nishida Kitarō (1870-1945) and Watsuji Tetsurō (1889-1960) respectively, the "self as place" and the "geocultural environment". In this way, we will show how a way of understanding the self as something situated and relational emerges that allows us to think of an ecological-communitarian way of being.
\end{abstract}

Keywords: Japanese philosophy, Nishida, Watsuji, place, milieu, basho, füdo, intimacy

1 El presente escrito está basado en la ponencia presentada en el Seminario International de Filosofía japonesa Towards the Ecological-Communitarian Self, organizado por el Colegio de Filosofía de la Universidad Nacional Autónoma de México y celebrado del 25 al 29 de noviembre de 2019, en el marco del proyecto PIFFYL 02_006_2019 “Ontologías intersticiales”, cuya participación fue apoyada por la Fundación Japón (México). 


\section{Introducción}

S upongamos que queremos ilustrar la imagen del ser humano y del lugar que ocupa en el mundo, natural y social, que ha predominado en la filosofía occidental. Podríamos partir de las célebres y enigmáticas palabras con las que Protágoras de Abdera habría iniciado su escrito $\alpha \dot{\lambda} \eta \dot{\theta} \theta 1 \alpha$ Aletheia (Verdad): " "De todas las cosas el hombre es medida, de las que son, como son, y de las que no son, como no son" (DK 80B1). ${ }^{3}$ O bien, podríamos partir de uno de los textos más representativos del Renacimiento, Oratio de hominis dignitate (Discurso sobre la dignidad del hombre, ca. 1486), donde Giovanni Pico della Mirandola dice haber comprendido por qué el ser humano es el más feliz y digno de admiración entre los seres vivientes y, remitiéndose al Génesis bíblico y al Timeo, reproduce las palabras que Dios habría dirigido a Adán después de situarlo en el centro del mundo creado: "La naturaleza bien definida asignada a los demás seres está encerrada en las leyes que hemos fijado. Tú, que no estás encerrado en ningún límite, establecerás tu naturaleza sobre la base de tu arbitrio, que he depositado en tus manos" (Bausi, 2014: 11). Este pasaje sería representativo, además, por constituir una síntesis de dos grandes fuentes de la filosofía occidental, la tradición semítica y la griega, puesto que el filósofo italiano equipara la noción platónica del ser humano como ser intermedio entre los mundos sensible e inteligible con la noción bíblica del hombre creado a imagen de Dios. También podríamos recurrir a textos del idealismo alemán, cuya filosofía "progresa desde la base de la ontología clásica hacia el descubrimiento de la personalidad humana como centro de todo conocimiento y de toda actividad" (Benz, 2016: 35), y mostrar, por ejemplo, cómo el Yo fichteano establece el mundo, quizás a partir del popular escrito de Fichte (2000) Die Bestimmung des Menschen (La misión del hombre, 1800). Para dar cuenta de una forma típica de pensar las relaciones entre el individuo y la sociedad, quizás

2 O titulado según Sexto Empírico, Kataballontes Logoi (Discursos demoledores), ver Adversus Mathematicos 7.60 (Untersteiner, 2008: 23-24, quien traduce la proposición transmitida por Sexto, y que no tendría como fuente a Platón, así: "l'uomo è dominatore di tutte le esperienze, in relazione alla fenomenalità di quanto è reale e alla nessuna fenomenalità di quanto è privo di realtà", en una traducción aproximada, "el hombre es dominador de todas las experiencias, en relación con el carácter fenoménico de lo real y el no fenoménico de lo que carece de realidad"). En adelante, la traducción al español de los pasajes citados, si no se indica lo contrario, es mía..

3 Para el texto griego y la traducción al alemán de Diels-Kranz 1959-1960: 262-263, de ahora en adelante DK. La traducción de Álvaro Vallejo Campos de la versión del Teeteto de Platón dice así: "el hombre es medida de todas las cosas, tanto del ser de las que son, como del no ser de las que no son" (152a). 
podría esgrimirse la defensa de la individualidad de John Stuart Mill en On Liberty (Sobre la libertad, 1859), donde ésta se presenta como el desarrollo más pleno de nuestra personalidad y, por tanto, la mejor forma de obtener la felicidad individual y el bienestar social. De este tratado, se podría citar el argumento de que al ser cada individuo libre de decidir por sí mismo lo que más le conviene, la coerción social, es decir, la interferencia en la libertad individual, sólo estaría justificada para evitar que se causen daños a los demás.

De ser el caso, seguramente se nos ocurrirían otros muchos escritos seminales como estos, que han ejercido una gran influencia y han contribuido a sentar las bases del antropocentrismo, el énfasis en el yo subjetivo, o el individualismo que sin duda nos vienen en mente para caracterizar la visión del ser humano que ha predominado en la mentalidad o el imaginario occidental. Sin embargo, se podría objetar que cada uno de los textos mencionados pertenece a un contexto distinto, expresa una cosmovisión diversa, mediante un enfoque y una terminología propia (por ejemplo,

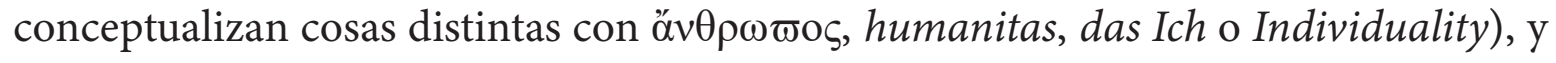
responden a un problema o una controversia diferente. Otra posible objeción sería que, junto a estas obras, también hallaríamos otros muchos escritos filosóficos occidentales, quizás de autores menos ampliamente leídos o de corrientes marginales o no hegemónicas, en los que se defiende lo contrario. De ahí que el mero intento de hablar de una forma de pensar o del repertorio de elementos simbólicos y conceptuales de una tradición tan variada, cambiante y compleja como la occidental constituya una generalización y una abstracción excesivas.

¿Y si tratamos de ilustrar mediante ejemplos tomados de distintas tradiciones de pensamiento asiáticas una mentalidad o un imaginario, por así decir, cosmocéntrico, relacional o comunal? Se nos podrían presentar las mismas objeciones. Y, con todo, nuestro interés aquí es reflexionar a partir de ciertos textos filosóficos japoneses en los que el ser humano es concebido como indisociable de la comunidad y del ambiente cultural y natural en que vive y le constituye, por tanto, como ser situado e interdependiente. Nuestro propósito es servirnos de fuentes de la filosofía japonesa para pensar un sí mismo ecológico-comunitario. La expresión "ecológico-comunitario" reúne en una sola unidad las dos dimensiones, natural y social, que atañen a dos de los retos más cruciales que debemos afrontar en nuestro tiempo: la emergencia climática y la fractura social. Y una forma de no eludir la tarea urgente de pensar en cómo responder a estos retos sería aportar una perspectiva filosófica que conciba al yo en un sentido menos individualista y al ser humano desde el lugar, relativo y relacional, que ocupa en el 
mundo. Para ello, la filosofía japonesa nos ofrece recursos conceptuales valiosos, por lo que partimos de la idea de que introducir en la reflexión filosófica fuentes procedentes de las tradiciones de pensamiento extraeuropeas puede contribuir a enriquecer la discusión y a que seamos capaces de diagnosticar y comprender mejor los problemas que nos afectan globalmente, así como proponer alternativas.

No obstante, queda la cuestión metodológica de cómo plantear dichas alternativas sin incurrir en la contraposición fácil entre un pensamiento occidental marcadamente antropocéntrico, que valora altamente el individualismo y gira en torno al yo subjetivo frente a un pensamiento asiático que habría privilegiado el aspecto comunal, relacional y cosmocéntrico (o quizás mejor, en otros términos "ecocéntrico") a la hora de pensar el lugar y las relaciones que el ser humano establece en los ámbitos natural y social. Un marco teórico adecuado para nuestro propósito lo ofrece Thomas P. Kasulis en su obra Intimacy or Integrity. Philosophy and Cultural Difference, publicado en 2002 a partir de las Gilbert Ryle Lectures que impartió el filósofo estadounidense en 1998. Fruto de su dilatada trayectoria como investigador y docente de filosofía comparada y asiática, Kasulis plantea en esa obra un paradigma interpretativo que permite identificar ciertas notas dominantes en el pensamiento desarrollado en diversas culturas y reconocer, siguiendo un modelo heurístico, patrones de relación sin caer en discursos esencialistas o etnocéntricos. En uno de sus últimos trabajos, Engaging Japanese Philosophy: A Short History (2017), ${ }^{4}$ aplica dicho paradigma al estudio de una serie de filósofos —esto es, Kūkai, Dōgen, Shinran, Norinaga, Sorai, Nishida y Watsuji- que elige como representativos de varias corrientes de pensamiento: budistas, confucianas, sintoístas y de la filosofía académica moderna, a través de las cuales recorre la historia de la filosofía japonesa. La obra en cuestión se propone además, como un intento de animar a los lectores a que interactúen con la filosofía japonesa, que no se limiten a aprender de ella como si de un objeto de estudio ajeno se tratara, sino que se atrevan a pensar junto a los filósofos los problemas que les preocuparon y a unirse a ellos en su intento de arrojar luz sobre las preguntas que formularon y las respuestas que ofrecieron. Con la implicación y complicidad del lector, Kasulis logra en cierto modo devolver a la filosofía una dimensión práctica que a menudo se olvida en la academia y que está en sintonía con la orientación de la "intimidad" que describe en su obra y define el filosofar de los autores japoneses que examina. De nuevo, cabe resaltar que hallaríamos ejemplos de esta orientación del pensamiento

4 Para la traducción al español, ver Kasulis (2019). 
en la forma de entender y practicar la filosofía en autores de otros lugares, también en filósofos occidentales. Desde esta perspectiva, al comparar o contraponer culturas y tradiciones distintas, no se trata de afirmar la superioridad de una sobre otra, sino de poner de manifiesto cómo nuestra manera de entender las relaciones con eso que llamamos naturaleza, con los demás e incluso con nosotros mismos, está en gran parte condicionada por la cultura, la tradición, y el modo en que la va construyendo el estudio bibliográfico y crítico de la historia, sus fuentes y los cánones derivados de dicho estudio.

El tomar en consideración este condicionamiento nos puede ayudar a comprender la diversidad cultural y presumiblemente también a pensar a partir de orientaciones distintas a las que estamos acostumbrados y cuya validez aceptamos acríticamente. Asimismo, este reconocimiento podría llevarnos a emprender una revisión historiográfica que ilumine las zonas que han quedado fuera de foco o que, con la apertura del objetivo, amplíe la profundidad del campo. De este modo, quizá no estaríamos lejos de un Protágoras, cuya línea de pensamiento ha sido comparada con la crítica de la concepción antropomórfica de los dioses de Jenófanes de Colofón en la medida en que ambos habrían partido de la observación empírica de la diversidad cultural para inferir que estamos obligados a adoptar una perspectiva esencialmente humana (Van Berkel, 2013: 61). ${ }^{5}$ Desde luego, compartiríamos con Pico della Mirandola la voluntad inclusiva, en su caso, diversas tradiciones filosóficas y teológicas antiguas, desde la mosaica a la socrática, la mistérica, pitagórica, caldea o la sabiduría cabalista y árabe, bajo la convicción de que cada una puede contribuir al logro de la verdad (Bausi, 2014: xxv). Siguiendo con los ejemplos citados antes, es bien conocido el interés entre los románticos alemanes por las filosofías índicas o por recuperar la tradición mística renana, e incluso Mill menciona en su tratado, aún con reservas, la filosofía china. Si bien considera a China estacionaria, y atribuye el que no progrese a su éxito en igualar a todas las personas y gobernar sus pensamientos y conductas mediante las mismas máximas y normas, se refiere a ella como "una nación de mucho talento y, en algunos aspectos, incluso sabiduría, debido a la rara buena fortuna de haber recibido

5 De este modo, Tazuko A. Van Berkel (2013) interpreta la posición de Protágoras más como un perspectivismo que como un relativismo, es decir, como una metáfora que indicaría que no podemos evitar ver las cosas desde una perspectiva de primera persona, como humanos no podemos ver el mundo sino en términos de la medida humana. La autora sugiere que quizás tanto Jenófanes como Protágoras reflexionan sobre la condición humana en sentido genérico como reacción a la tradición sapiencial arcaica y las cosmologías presocráticas que tomaban como punto de partida para pensar la moral la realidad o el ser. 
en un período temprano un conjunto de costumbres particularmente bueno, obra, en cierta medida, de hombres a quienes incluso los europeos más ilustrados deben conceder, bajo ciertas limitaciones, el título de sabios y filósofos” (Mill, 2003: 136-137).

A fin de contribuir a ampliar nuestra mirada sobre el tema que nos ocupa con la inclusión de fuentes de la filosofía japonesa, a continuación presentamos brevemente el paradigma de la intimidad y de la integridad, según la caracterización propuesta por Kasulis, para después analizar cómo, desde el paradigma de la intimidad, se puede articular lo que hemos denominado "yo relacional" a partir de las creaciones conceptuales del "yo como lugar" de Nishida Kitarō (1870-1945) y el "ambiente geocultural" de Watsuji Tetsurō (1889-1960).

\section{¿Intimidad o integridad?}

En su deseo de hacer comprensible la filosofía japonesa a un público amplio, no necesariamente familiarizado con el pensamiento o la cultura de Japón, Thomas P. Kasulis se sirve a menudo de la contraposición entre las dos orientaciones culturales, "intimidad" e "integridad", que, como se ha dicho, expone ampliamente en su obra Intimacy or Integrity. Para llevar la discusión teórica a un terreno más cercano y concreto para sus lectores u oyentes, en ocasiones, formula las siguientes preguntas:

¿Quién conoce mejor la arcilla: (A) un geólogo o (B) un alfarero?

¿Quién conoce mejor las palabras: (A) un filólogo o (B) un poeta?

¿Quién conoce mejor la luz: (A) un físico o (B) un fotógrafo?

¿Quién conoce mejor la respiración: (A) un neumólogo o (B) un meditador?

¿Quién conoce mejor las familias: (A) un sociólogo o (B) un consejero

familiar? (Kasulis, 2019: 34).

Como si de un test se tratara, añade que si eres una persona que tiende a preferir la respuesta $\mathrm{B}$, respondes como la mayoría de los filósofos japoneses, es decir, tu orientación cultural es la del conocimiento por implicación, el cual corresponde al paradigma de la intimidad. En cambio, si has contestado la mayoría de veces A, tu orientación cultural tiene más que ver con la de la integridad, a saber, la que es propia de la búsqueda de un conocimiento objetivo mediante el distanciamiento y que cobró relevancia en la filosofía occidental particularmente a partir del siglo XVII. 
Todas las actividades que llevan a cabo las personas mencionadas en las preguntas citadas nos resultan conocidas, aunque no las cultivemos ni dispongamos de los conocimientos necesarios para hacerlo de manera profesional o experta, ya sea nuestro lugar de origen occidental o asiático. Las profesiones que aparecen en la categoría A claramente requieren de una instrucción y titulación universitaria, de unos conocimientos adquiridos sobre la base de la manera en que se ha entendido y practicado la ciencia según el modelo occidental y que, por razones históricas, a menudo no debido al mero intercambio del saber sino al dominio colonial, se ha generalizado ampliamente en el resto del mundo. Las actividades que desempeñan las personas clasificadas como $\mathrm{B}$, en cambio, requieren un tipo de habilidad que quizás denominaríamos un saber hacer o sabiduría práctica (algo que, cabría matizar, hasta cierto punto, no necesariamente deberíamos descartar en el ejercicio de las profesiones clasificadas como A; o incluso las dos orientaciones podrían darse en una misma persona, quizás, en diferentes situaciones). En todo caso, Kasulis pone el acento en el tipo de conocimiento que A o B obtienen de algo en particular que es central en su actividad (arcilla, palabras, luz, respiración, familias). Si nos detenemos en el primer ejemplo, la arcilla, es obvio que un alfarero, aunque carezca de estudios de geología, tras años de oficio, conoce a la perfección el material que moldea. Digamos que un conocimiento de ese tipo y el modo de adquirirlo ha interesado particularmente a los filósofos japoneses. Así, mediante esta estrategia discursiva, que nos obliga a recurrir a nuestra experiencia para poder posicionarnos, nos encontramos en disposición de abordar nuestro objeto de estudio y de superar la extrañeza que, de entrada, pudieran causarnos los textos filosóficos japoneses.

Teniendo esto en mente se entenderá mejor la siguiente caracterización del paradigma de la intimidad que propone Kasulis (2002: 24, 32):

1. La intimidad es objetiva, pero más personal que pública.

2. En una relación íntima, el yo y el otro se copertenecen de una manera que no permite distinguir netamente entre los dos.

3. El conocimiento íntimo posee una dimensión afectiva.

4. La intimidad es somática, así como psicológica.

5. El fundamento de la intimidad por lo general no es autoconsciente, reflexivo, ni arroja luz sobre sí mismo.

Intimidad implica, por tanto, inseparabilidad, copertenencia, compartir. Se trata de establecer un tipo de relación especial, de estar íntimamente relacionados, de poseer 
un conocimiento íntimo de lo que queremos conocer, de intimar, como la relación de complicidad que sólo mantenemos con nuestros allegados más íntimos, con quien nos une un estrecho vínculo. Como nos recuerda Kasulis, la palabra procede del latín intimus, que significa "lo que es más interior" o "un amigo cercano", mientras que el verbo intimare significa "dar a conocer". Reuniendo los dos sentidos, Kasulis se refiere al sentido original de intimidad como un "dar a conocer a un amigo cercano lo más íntimo". Por consiguiente, una relación íntima implica que no podemos entender los elementos que se encuentran relacionados uno sin el otro, pues, aunque los separemos mediante la abstracción, siguen entrelazados, es decir, comparten cualidades esenciales.

En cambio, la palabra integridad procede del latín integritas, vocablo relacionado con integer, esto es, una totalidad indivisible. Kasulis también lo relaciona con in y tangere, es decir, no tocar. Lo que posee integridad permanece intacto, incorrupto, puro. Así, integridad se refiere a una totalidad indivisible e inviolable. De acuerdo con esto, el paradigma de la integridad subraya los siguientes rasgos:

1. Objetividad en cuanto verificabilidad pública.

2. Enfatiza las relaciones externas sobre las internas.

3. El conocimiento como idealmente libre de afectos.

4. Distingue lo intelectual y psicológico de lo somático.

5. Se refiere a un conocimiento reflexivo y autoconsciente de sus propios fundamentos (Kasulis, 2002: 25).

Tras estas dos orientaciones reside un modo distinto de relacionarse. Si vamos a examinar una forma de concebir el yo, o sí mismo, que hemos descrito como relacional, conviene detenerse brevemente en esta idea que, aunque relativamente sencilla, resulta sumamente reveladora cuando se aplica no sólo al campo epistemológico, sino también al ético-político o al estético. Pensemos en dos cosas, A y B, relacionadas. Si las pensamos como dos objetos independientes, A y B existen en su integridad antes de entrar en relación y después de establecer la relación una tercera cosa $(R)$ las mantiene conectadas entre sí. Si desaparece R, A y B siguen manteniendo su individualidad íntegramente. Kasulis se refiere a este tipo de conexión como una relación "externa" porque el vínculo existe como algo ajeno al carácter intrínseco de las dos cosas relacionadas. Pensemos, por ejemplo, en un puente construido sobre el Danubio para unir las dos poblaciones medievales de Buda y Pest, en una sola ciudad. Sin el 
vínculo añadido que constituye el puente y, por tanto, sin un modo de cruzar de una orilla a otra, la distinción entre ambas poblaciones se habría mantenido.

Por el contrario, si entre A y B se mantiene una relación "interna", no son independientes sino interdependientes, una es inherente a la otra. Para estar completos, y mantener su integridad, A necesita de B, y a la inversa. La imagen de la intersección de dos conjuntos que se emplea en las matemáticas puede ser ilustrativa: los elementos que quedan en el medio pertenecen a los dos conjuntos, los comparten; R en este caso sería la relación que se da al superponerlos. Si la relación se disolviera, A y B dejarían de ser lo que son, o perderían parte de lo que eran. Por ejemplo, si un pintor deja de mezclar los pigmentos con aceite dejará de tener pintura al óleo, o si separamos el amarillo del azul, dejamos de obtener el verde. Si aplicamos esto a la manera indisociable de concebir el cuerpo y la mente, o la mente y los sentimientos, en la filosofía japonesa tradicional, nos resultarán sorprendentes los intentos por determinar qué vincula a ambos en buena parte de la filosofía occidental, la cual comienza con el supuesto de que en cuanto entidades distintas son separables. Conviene insistir una vez más en que las dos orientaciones descritas no son exclusivas de ninguna tradición cultural o filosófica, pero sí que han tendido a privilegiar una u otra, de modo que han predominado de manera más ostensible en unas que en otras.

\section{El yo situado y relacional}

Desde estas premisas, pasemos a continuación a abordar la cuestión del yo relacional a partir de dos conceptos que hallamos en la filosofía japonesa moderna, y que, como veremos, están vinculados a concepciones del pensamiento asiático premoderno en las que prevalece la orientación de la intimidad. El primer concepto es la noción formulada por Nishida Kitarō de basho 場所, la cual marca un punto de inflexión en el pensamiento del filósofo. A lo largo de su vida, Nishida intentó construir su filosofía de una manera que podría calificarse de sistemática; sin embargo, crítico incansable de su propio trabajo, nunca pareció satisfecho con su propio modo de explicación y reformuló continuamente sus ideas acuñando nuevos términos. La noción aparece por primera vez en la colección de ensayos titulada Del que actúa al que ve (Hataraku mono kara miru mono e 働くものから見る物へ, 1927) y Nishida va precisando su significado en sus escritos posteriores. 
En esa etapa de su camino filosófico, Nishida trató de articular su idea de que la experiencia original del mundo debe ser de unidad, previa a la diferenciación entre sujeto y objeto, ser y nada, y de la cual surge cada pensamiento y fenómeno como una determinación particular de ello. Denomina "lugar" a este tipo de campo original:

Conocer simplemente significa que la experiencia vivida se forma a sí misma dentro de sí. En el lugar de la experiencia vivida se constituye la relación de contraposición entre forma y materia. Ahí, donde eso que siendo en sí mismo nada contiene el ser infinito - a saber, el "yo" verdadero- se va reflejando infinitamente dentro de sí, se constituye la contraposición entre sujeto y objeto. Tal lugar no puede ser descrito como idéntico o diferente; no puede decirse que sea ser o nada [...] La verdadera forma de la forma debe ser el lugar de las formas. (Nishida, 2016: 675-676)

Nishida parece pensar en basho como un lugar o espacio, no en un sentido físico sino lógico, gnoseológico, ontológico e incluso religioso. Podría entenderse como un trasfondo, un campo contextual, o quizás, tomando prestada la terminología del misticismo especulativo, un "fondo sin fondo" (por ejemplo, el grunt âne grunt eckhartiano), donde tienen lugar los juicios, las distinciones sujeto-objeto que producen el conocimiento, las relaciones interpersonales y los fenómenos.

La idea de basho se le ocurrió a Nishida cuando analizaba la noción de $\chi \omega ́ \rho \alpha$ chōra tal como aparece en el Timeo de Platón, ${ }^{6}$ donde leemos: "decimos que necesa-

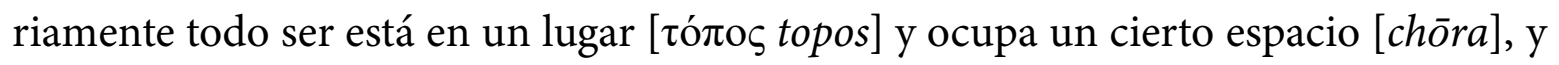
que lo que no está en algún lugar en la tierra o en el cielo no existe" (Platón, Timeo: 52b). Como es sabido, Platón describe chōra como una matriz o receptáculo que recibe todas las cosas sin con ello adoptar la forma de las cosas que contiene, si bien es modificado y moldeado por las cosas haciendo que parezca distinto en diferentes momentos. Por tanto, la idea de chōra expresa una cierta ambigüedad o indefinición, ya que no es ni subjetiva ni objetiva, ni idea ni cosa, ni forma ni copia: ni modelo inteligible e inmutable, ni imagen del modelo, que deviene y es visible (49); como no pertenece a ninguno de los dos géneros (inteligible y sensible), Platón necesita diferenciar conceptualmente tres géneros (50d) y describe chōra como el tercer género o tercera clase (52a). Al carecer de una identidad propia, chōra permanece indeter-

6 Sobre el basho de Nishida y chōra, ver Tremblay (2007: 59-72) y Krummel (2015: 191-207). Sobre otras interpretaciones de chōra a partir de Platón, ver Derrida, Chōra (1993) y Sallis (1999). 
minado, sin carácter, sin forma y sólo puede ser intuido o, como Platón lo expresa, captado por medio del razonamiento espurio o "bastardo" (52b), no por un razonamiento exacto y verdadero propio del conocimiento del ser.

Interesado en el aspecto lógico de basho, Nishida procedió a analizar desde esta perspectiva la estructura lógica de la forma de juicio y llamó a su teoría una "lógica de lugar" (basho no ronri 場所の論理). Es una lógica predicativa o topológica en la que el plano del sujeto queda en cierto modo subsumido en el plano del predicado. La idea básicamente sería que cualquier juicio surge necesariamente de un basho particular, es decir, un lugar particular, pero dado que puede haber una pluralidad de verdades y contextos, tuvo que explicar cómo se interrelacionan dichos contextos.

La "lógica del basho" de Nishida es un sistema complejo que siempre cambia y está sujeto a revisión, pero podemos resumir brevemente su núcleo para sugerir cómo se desarrolló su línea de pensamiento. Jacynthe Tremblay (2007: 48-49) se sirve de la imagen muy gráfica, la de las matrioshka o muñecas rusas, para facilitar la comprensión de la estructura englobante de esta lógica. Una forma en que Nishida formuló este sistema fue en términos de tres basho: el del ser ( $u$ no basho 有の場所), la nada relativa (sōtai mu no basho 相対無の場所) y la nada absoluta (zettai mu no basho 絶対無の場所). Estos corresponden aproximadamente a los campos de juicio del empirismo (que pretende expresar pura objetividad con una declaración sobre algo que es y que omite o neutraliza el observador, por ejemplo, si digo "el sol brilla afuera"), del idealismo (en cuyo juicio subjetivo el observador entra en juego, por ejemplo, "yo veo a través de la ventana que el sol brilla afuera y me siento feliz"), y, finalmente, lo que Nishida llamó el campo de la "intuición activa", o también traducida como “intuición enactiva”, ( $(k o \bar{i} t e k i$ chokkan 行為的直観). Este último campo sería el más comprehensivo. Como hemos visto, el campo del juicio objetivo del empirismo, el cual normalmente adopta la ciencia, excluye el papel del sujeto sin cuya observación, advierte Nishida, no podríamos afirmar nada sobre algo, por lo que considera que el primer campo está comprendido (incluido dentro, como una muñeca rusa) en el de la autoconciencia, un campo de juicios sobre uno mismo y su relación con los objetos de la experiencia, al que llama de la nada relativa, en referencia al yo anulado o reducido a nada en los juicios empíricos. Con todo, a diferencia del idealismo, Nishida no concibe el yo como sustancia, sujeto o ego trascendental, sino como agente. El basho último es el campo donde tiene lugar la acción, la "intuición activa" (en

7 Sobre este concepto, ver Cestari (1998) y Maraldo (2017). 
nuestro ejemplo, el mero acto de ver, brillar, sentir calor o felicidad, etcétera). Así, el basho del idealismo que ve al yo como sujeto y objeto está comprendido en un tercer basho, el de la nada absoluta. La expresión "intuición activa” reúne el aspecto pasivo asociado con la intuición, es decir, nuestra recepción de información sobre el mundo, y el aspecto activo de nuestra participación en dicho mundo. En este campo, la intuición y la acción son inseparables como dos polos de un solo proceso. Remite a un proceso, no a una cosa, por lo que nunca puede ser el sujeto o el objeto en sí mismo y por eso es denominado "nada absoluta". Es tan imposible de definir o conceptualizar, pues se convertiría en una cosa, un objeto de la conciencia; sería como intentar pensar la muñeca rusa más grande posible, en la imagen de Tremblay, o un círculo infinito en una de las imágenes que emplea Nishida. Sólo se puede decir que es el lugar donde se sitúan todas las cosas y se relacionan entre sí, donde tiene lugar el mundo de la naturaleza, de los individuos y del conocimiento, pues es donde el "yo verdadero" (shin no jiko 真の自己) conoce las cosas en sí mismas.

En su trabajo posterior, Nishida ve el basho como el "lugar" de formación de la historia. El mundo histórico está situado en el basho de la nada absoluta, por así decir, como su contenido. El "lugar" da forma a un mundo histórico que no es el mundo natural de las cosas, ni el mundo de la vida de la conciencia del sí mismo, sino el mundo de la cultura. En basho, el mundo se determina a sí mismo como un mundo sociohistórico. Reflejando a todos los individuos y su forma de ser mutuamente determinante dentro de sí mismo, basho es un lugar en el que todos los seres vivos y no vivos llegan al ser. Es un "lugar" de existencia relacional: "Lo que se considera el 'yo individual' no es más que una cosa que se puede pensar como la determinación individual del mundo que se determina a sí misma" (Nishida, 1965a: 203). El mundo, por tanto, puede entenderse como el lugar de la intuición activa mencionado antes, lo que se comprenderá mejor con la analogía que emplea el propio Nishida de una representación teatral (Nishida, 1965b: 290). Mientras que el basho de ser equivaldría al público, el observador distante que ve una obra de teatro con objetividad, los actores, que interpretan un personaje y se involucran de manera activa y subjetiva en la ejecución de la obra, corresponderían al basho de la nada relativa. Sin embargo, en la medida en que los actores sólo se implican en la obra desde el punto de vista del papel que representan, no experimentan el despliegue total de la obra. Análogamente, los seres humanos participamos en el mundo e incluso podemos analizarlo de manera distanciada, como sugiere la orientación de la integridad mencionada antes, pero la obra de teatro que se representa no sería sino el basho de la nada absoluta o 
la intuición activa, una posición que incluye la experiencia y la participación en el desarrollo de la totalidad, la unidad o la no dualidad, de sujeto y objeto.

Esta superación de la dicotomía sujeto-objeto y centralidad de la acción o el proceso está en consonancia con lo que Nishida escribió en el Prefacio de la primera edición de su primer libro, Indagación del bien (Zen no kenkyū, 1911): "Al pasar el tiempo, llegué a comprender que la experiencia existe no porque haya un individuo, sino que un individuo existe porque existe la experiencia" (Nishida, 1995: 34). Esta afirmación no implica necesariamente una negación ontológica de la individualidad como tal. Más bien significa que el individuo surge en la experiencia; por lo tanto, es algo mutable, no algo fijo más allá del flujo experiencial. De esta manera, Nishida invierte sutilmente la forma habitual de pensar la relación entre individuo y experiencia, como cuando decimos "he tenido una experiencia". Piensa en la experiencia como un campo de autoestructuración que da forma tanto a lo que pensamos como "yo" o "sí mismo", como a lo que pensamos como mundo. Es decir, Nishida plantea que el yo no es lo que constituye la experiencia, sino que la experiencia constituye el yo. Tampoco cabe pensar que la realidad, como algo externo a mí, me proporciona los datos de lo que experimento, sino que la realidad es la forma en que la experiencia toma forma. Por esta razón, con la noción de basho da cuenta de este campo autoconsciente y autoformativo.

Pasemos ahora al siguiente concepto, el que da título al ensayo de Watsuji Tetsurō, $F \bar{d}$ do 風土 (1935). Watsuji se formó en la filosofía occidental y estudió en particular la hermenéutica y la fenomenología. De hecho, se dio a conocer en Japón con sus obras sobre Nietzsche y Kierkegaard. Sin embargo, también se interesó en las filosofías asiáticas y no dudó en tomar nociones del pensamiento confuciano y budista. Ambas fuentes confluyen en el proyecto filosófico de Watsuji, el cual se suele interpretar como un intento de repensar las relaciones entre la individualidad, la vida ética y el mundo natural. La idea central de su ensayo es de especial relevancia para la cuestión que abordamos aquí: la naturaleza tal como se experimenta y se vive es parte de la estructura misma de la existencia humana, de modo que el yo se halla inmerso en esta dimensión de la naturaleza y en continuidad con ella.

De esta idea se puede extraer la conclusión de que el ser humano sólo puede ser lo que es a lo largo de su vida, al incorporar y dar expresión cultural a una región de la naturaleza, y que, a su vez, dicha región particular de la naturaleza sólo puede ser completamente lo que es siendo parte y desplegándose a través del mundo de la cultura humana. Esta bidireccionalidad constituye füdo, traducido al castellano 
como "ambientalidad" o "clima y paisaje", al italiano como Vento e terra (literalmente, pues los dos sinogramas que componen la palabra significan "viento" y "tierra"), al inglés como climate and culture y milieu (que viene del francés, mi significa "medio" y lieu significa "lugar"), y que recientemente David Johnson ha parafraseado como el medio ambiente geocultural, al que nos abrimos y al cual pertenecemos. ${ }^{8} \mathrm{El}$ hecho de que el concepto no se refiera meramente a la naturaleza ni se pueda separar de lo humano (en este sentido, adelantando quizás la tesis central de los teóricos del Antropoceno sobre el impacto de la actividad humana sobre los ecosistemas terrestres) lo deja claro el autor en el Prólogo a la obra, donde leemos:

El objeto de esta obra es resaltar la importancia de la ambientalidad — clima y paisaje- como elemento estructural de la existencia humana. No se trata solamente del influjo del medio ambiente en el ser humano, porque lo que se entiende habitualmente por medio ambiente es algo derivado, que tiene su fundamento en lo que aquí llamo ambientalidad climático-paisajística como característica de la vida humana.

Cuando hablamos de relación entre el medio ambiente y la vida humana, ésta se encuentra ya objetivada. Dicha reflexión parece pensar la relación entre dos objetos, en vez de ocuparse de la existencia humana en su carácter de subjetividad, que es lo que aquí me interesa.

A lo largo de esta obra se aludirá a menudo al problema de las formas que revisten clima y paisaje, pero no como mero entorno físico, sino como expresión existencial del sujeto humano. (Watsuji, 2006: 17)

Watsuji distingue entre füdo y el medio ambiente natural (shizen kankyō 自然環 境), pues este último equivaldría a una abstracción que convierte la naturaleza en un objeto analizable, en la línea podríamos añadir de la orientación de la integridad, mientras que el primero consiste en fenómenos que presuponen la existencia del sujeto humano que los vive, percibe e interpreta, claramente en la línea de la orientación de la intimidad. Watsuji descarta el determinismo ambiental y habla de la libertad del sujeto humano en reciprocidad con el entorno natural.

Tras el pasaje citado arriba, en el Prólogo de Fūdo, Watsuji explica que Sein und Zeit (El ser y el tiempo, 1927) de Heidegger inspiró su trabajo. Watsuji leyó en Berlín

8 Véanse las traducciones de Watsuji (1960, 1988, 2011, 2006, 2015), así como el estudio de David Johnson (2019). 
el libro recién publicado de Heidegger con grandes expectativas sobre cómo utilizaría la fenomenología de una nueva manera para abordar las cualidades de la existencia humana. Sin embargo, decepcionado, critica a Heidegger por no haber dado suficiente importancia al espacio en su obra, lo cual se refiere principalmente a Sein und Zeit, ya que la cuestión del espacio no está ausente de las obras de Heidegger, pero parece ser de importancia secundaria antes la década de 1950. De hecho, Heidegger escribe (§5):

mostraremos que aquello desde lo cual el "ser ahî" en general comprende e interpreta, aunque no expresamente, lo que se dice "ser", es el tiempo. Éste tiene que sacarse a la luz y concebirse como el genuino horizonte de toda comprensión y de toda interpretación del ser. Para hacerlo evidente así, se ha de menester de una explanación original del tiempo como horizonte de la comprensión del ser, partiendo de la temporalidad como ser del "ser ahí" que comprende el ser. (Heidegger, 1996: 27)

Watsuji ve en Heidegger un desequilibrio que resulta de priorizar la temporalidad sobre la espacialidad, lo mental sobre el cuerpo y enfatizar la naturaleza individual del ser humano. Para el filósofo japonés, la estructura de la existencia humana no es menos espacial que temporal y, en consecuencia, no se puede subordinar la espacialidad a la temporalidad. Watsuji también afirma que la existencia humana no es menos social que individual y, por lo tanto, uno debe tener en cuenta la dualidad socialindividual del ser humano, término que, en japonés, se expresa como ningen 人間. $\mathrm{El}$ primer componente se refiere a "individuo" $人$ y el segundo tiene una connotación espacial 間 (leído ken es la longitud de un tatami; leído ma es, entre otras cosas, una habitación en una casa, y leído aida es un intervalo o relación entre dos cosas, eventos o personas). Este segundo sinograma que compone la palabra "ser humano" agrega, por tanto, la connotación de que las personas se vuelven humanas cuando viven entre otras. ${ }^{9}$ Según Watsuji, en su descripción del "ser ahí" (Dasein), Heidegger, pese a que introduce la idea del "ser con" (Mitsein), es decir, "ser con otros", no ofrece una explicación satisfactoria de qué significa ser un ser humano en el mundo, pues, en su

9 Resulta interesante, además, que el significado de este carácter en China remitía al tiempo, mientras que en Japón adquirió el sentido espacial. Etimológicamente es una combinación del sinograma "puerta" 門 y "luna” 月, evocando la imagen poética de la luz de la luna filtrada a través de un panel deslizante semiabierto al exterior. Más tarde, el componente "luna” del carácter original 閒 fue reemplazado por el "sol” 日. 
opinión, pasa por alto la influencia de la cultura y las vastas conexiones de red que nos hacen lo que somos, individuos inmersos en el espacio/tiempo de un mundo, junto a otros. Para Watsuji, no somos individuos solitarios que conviven junto a otros individuos también solitarios, sino seres de lo que denomina "interidad" o "ser entre" (aidagara 間柄), estar en medio. El énfasis dado a los aspectos espacial y social del ser humano lo lleva a dedicar su ensayo a la conceptualización de la relación de una sociedad con su entorno ( $f \bar{u} d o$ ), así como el sentido y orientación de esta relación, a lo que denomina (fūdosei 風土性) y define como momento estructural de la existencia humana.

Curiosamente, para traducir el verbo “ser" (sonzai suru 存在 する) y “existencia" (sonzai 存在), la filosofía japonesa ${ }^{10}$ usa dos sinogramas y uno de ellos incorpora originalmente la idea del lugar $y$, por tanto, la espacialidad: $z a i$, que comprende el radical "tierra" (土), significa "estar en un lugar", o "el lugar del ser". Teniendo en cuenta estas particularidades de la lengua japonesa, Augustine Berque, quien ha estudiado en profundidad tanto la obra de Watsuji como las concepciones del espacio y la naturaleza en Japón, reconoce que para un japonés resulta difícil imaginar al ser humano o la existencia humana (ningen sonzai 人間存在) sin hacerlo espacialmente, y concluye que en japonés "el ser no trasciende el mundo" (Berque, 1998: 247).

\section{Conclusión}

Extraeremos, a continuación, algunas conclusiones de lasideas expuestas en el apartado anterior a la luz de la orientación de la intimidad propuesta por Kasulis y en conexión con la cultura japonesa. Como hemos visto, el concepto nishidiano de basho no sólo significa dónde o cómo tiene lugar una experiencia o un juicio en la experiencia, sino que también sitúa una experiencia al establecer un punto de vista o un contexto envolvente para la forma en que se juzga algo, y sitúa cada juicio en términos de su contexto experiencial más amplio. De su estudio de la filosofía occidental le llamó especialmente la atención la polarización entre sistemas filosóficos idealista o empírico, los ámbitos mental o material, o los conceptos de subjetivo y objetivo. En lugar de decantarse por uno de los polos o elaborar una síntesis de ambos, Nishida trató de definir un sistema

10 La palabra más común en japonés con el sentido de "ser" o "existir" es u 有 (también leído yū o a(ru) 有る), que originalmente significa "haber", pero también puede significar "estar situado (en)" o "estar contenido (dentro de)". 
lógico que proporcionara el fundamento de todas esas dicotomías. Nishida argumenta que hay algo más básico que el yo que constituye el yo, es decir, un proceso creativo y receptivo, una idea con una clara resonancia religiosa, ética o estética, que nos sitúa en la línea del conocimiento más bien personal, somático y afectivo que veíamos en la orientación de la intimidad. Este terreno inefable, el basho de la nada absoluta, es la base tanto para uno mismo como para el mundo empírico, tal como se conoce a través de la ciencia. En él, sujeto y objeto, interioridad subjetiva y ambiente externo se realizan determinándose como contradictorios. Negándose, el ambiente da forma al individuo, el cual emerge del ambiente y se separa de él; a su vez, el individuo da forma al ambiente, negándose, deja espacio al ambiente para ser, lo determina deslizándose en su fondo. Como en la orientación de la intimidad, uno no se entiende sin el otro, y el acento recae en el aspecto relacional y contextual.

En cuanto a füdo, conviene recordar que, con esta palabra, Watsuji intentó acuñar un equivalente espacial al concepto temporal de historicidad. En términos más generales, quería utilizar füdosei como base para una teoría fenomenológica de la humanidad (como lo revela el subtítulo de su libro "Un estudio del ser humano" y de ahí la palabra "Antropología" del título de la edición española de la obra) que complementaría Sein und Zeit de Heidegger como una mejor descripción del ser "en el mundo" de nuestra existencia. Para Watsuji, ser humano como ningen implica de entrada un sentido relacional, encarnado en el ser que incluye al individuo, la espacialidad de sus relaciones y los lugares y espacios geográficos/climatológicos y culturales en los que vive e interactúa. Füdo como "clima cultural" conceptualiza un "entre", un espacio intermedio entre la cultura y la civilización, por un lado, y la naturaleza, por otro. El pensamiento japonés premoderno no planteó esta dicotomía en términos tan excluyentes como vemos en gran medida en la filosofía occidental. Para Watsuji, como para buena parte de la filosofía japonesa, la civilización humana no puede ser vista como opuesta a la naturaleza, tal como la mente no puede ser vista como opuesta y separada del cuerpo. De nuevo podemos reconocer claramente la orientación de la intimidad. El "entre" es visto como un estándar de los seres humanos y de la cultura, pues indica que el sujeto siempre está vinculado al espacio. Füdo también destaca la diferencia entre las concepciones japonesas tradicionales y occidentales del yo. Mientras que, en la visión occidental moderna, el yo y el medio ambiente son por lo general términos contrapuestos, en Japón se consideran interrelacionados; el yo se funde con el medio ambiente al identificarse con patrones de la naturaleza que, sin embargo, están construidos culturalmente. 
Se puede decir, por tanto, que la "espacialidad" y la "relacionalidad" son ideas clave en estos dos filósofos japoneses. Ambos pensadores, Watsuji y Nishida, rechazaron el sujeto moderno, expresado de manera paradigmática en el cogito cartesiano autoinstituido y sustancial, y se centraron en la interrelación de este sujeto con su contexto (social, natural, histórico, cultural). Si bien esto significa una autodescentralización o deconstrucción del sujeto, han enfatizado que el ser humano siempre está en el mundo, como el In-der-Welt-Sein heideggeriano. Este tipo de yo, que es al mismo tiempo un no-yo (pues se autodetermina negándose), puede interpretarse como un proceso dialéctico en el que se autodetermina y es determinado en sus relaciones. Ambas dimensiones, individual y comunal, uno mismo y otros, el ser y el lugar de su ser, están co-implicadas y son necesarias. Ahora bien, cabe mencionar las implicaciones sociopolíticas del énfasis en esta dimensión locativa del ser. El hecho de que el yo sea siempre un yo ubicado o situado es muy importante para reconocerlo al enfrentar problemas globales como la paz, la justicia, los derechos humanos, el medio ambiente, etc. Sin embargo, la identificación del sujeto con su contexto puede considerarse como un riesgo de suprimir la alteridad del otro y subordinar al individuo al todo. $Y$ dado el período histórico en el que ambos pensadores vivieron y publicaron sus ideas, surge la cuestión de su apoyo a las ideologías totalitarias. Sin embargo, este problema es demasiado complejo para desarrollarse más aquí. En cambio, me gustaría referirme brevemente al trasfondo cultural budista y japonés que revelan basho y füdo.

En el pensamiento budista, por medio de la palabra sánscrita anātman, generalmente traducida como "no yo", se rebate la permanencia y sustancialidad del ätman hindú, una especie de alma que reside en el cuerpo, sería inmortal y transmigra en diferentes vidas hasta que logra su liberación. El yo, o sí mismo, desde el punto de vista budista (si bien la cuestión generó controversias entre las diferentes escuelas antiguas), tiene su propia identidad sólo en la medida en que podamos definir sus límites, pero, en realidad, carece de algo que no comparta o se superponga con otros. La identidad del yo sería convencional y provisional. No sólo está compuesto por una serie de agregados que se disuelven, tal vez dejando un residuo de conciencia, tras la muerte del individuo, sino que es producto de una serie de condicionamientos que le constituyen y siempre cambiante, por lo que más que una sustancia o un sujeto se trata de un proceso. Esto se aplica a la realidad en su conjunto, típicamente se representa con la imagen clásica de "la red de Indra": la realidad como una red interdependiente de procesos cambiantes, de acuerdo con la noción del "origen condicionado" de todos los fenómenos (en sánscrito, pratītya samutpāda). Cada fenómeno es au- 
toconsistente sólo en virtud de los condicionamientos que lo originan y constituyen. Desde la perspectiva budista, la identidad sustancial o independiente es una ilusión. Cada cosa que carece de sustancia está vacía y sólo se define por el flujo de procesos interdependientes que la configuran. El budismo ofrece una visión procesual del sí mismo y de la realidad fenomenal cercana al énfasis de Nishida y Watsuji en el trasfondo o espacio intermedio que hace posible la relación.

Por último, remitiré dos ejemplos ilustrativos con respecto al legado cultural japonés. Como hemos visto, para Nishida, el "yo" es una especie de lugar de experiencia, un lugar en que se ubican tanto lo lógico como lo sensorial. Esta idea se puede entender fácilmente examinando el idioma japonés. La frase que tomo prestada como ejemplo de Ueda Shizuteru (2004), "Escucho el sonido de una campana", en japonés, kane no oto ga kikoeru 鐘の音が聞こえる, dice literalmente “El sonido de la campana es oído" (161). Téngase en cuenta que no es necesario recurrir a un “yo”. Por supuesto, el japonés, como la lengua española, puede especificar el sujeto si es necesario, pero en el habla ordinaria no lo es. En este caso, el "yo" lógico emerge o surge de la experiencia de la resonancia de la campana y su resonancia se hace manifiesta.

El segundo ejemplo está tomado de Berque, quien argumenta que la visión occidental de la realidad debe mucho a la estructura básica de las lenguas europeas. Por ejemplo, la sustancia y los accidentes en metafísica corresponden al sujeto y al predicado en la lógica, mientras que la idea budista de que no existe una sustancia permanente, sino que todo es relacional y cambiante, está en sintonía con los rasgos básicos del idioma japonés. Para ilustrar esta idea, cita el siguiente haiku de Ōshi, que forma una oración completa y ordinaria:

\section{風鈴 の Fürin no (The windbell's)$$
\text { ちひさき音の chiisaki oto no (tiny chime:) }
$$$$
\text { 下にある shita ni iru (I am under) (en Berque 2016: 15) }
$$

En una traducción aproximada y más bien literal al español, el poema reza "el sonido mínimo de / las campanillas de viento: / estoy debajo". ${ }^{11}$ La poesía haiku, generalmente, incluye una palabra referida a una estación, aquí windbell o campanillas de

11 Nótese la alteración del orden de los dos primeros versos para el posesivo, es decir, que el sonido es de las campanillas de viento: en inglés lo pueden mantener gracias al genitivo sajón ('s). También se han añadido los artículos (en género y número, de los que carece el japonés), y se ha conjugado el verbo de manera personal, mientras que el japonés original correspondería a un verbo en infinitivo ("estar debajo"), si bien, el hecho de que el verbo sea iru y no aru sugiere que quien está situado debajo es un ser animado, humano o animal. 
viento (el primer componente de fürin es el viento), pues estas campanitas ligeras se cuelgan en verano de una rama en el jardín y suenan con una ráfaga de viento, lo que favorece que el lector u oyente sienta la brisa sinestésicamente. Lo sorprendente es que no hay un sujeto en la oración, sólo una escena, un ambiente, un conjunto particular de circunstancias, un entorno, o para decirlo con Watsuji, un füdo. Sin embargo, nótese que, en la traducción del haiku al inglés, para dar cuenta de la acción que transcurre en el "lugar" (el estar debajo de la campana) se ha añadido (explicitado y con ello, distinguido) un "yo" (I).

\section{Referencias bibliográficas}

Bausi, Francesco (Ed.). (2014). Discorso sulla dignità dell'uomo, Giovanni Pico della Mirandola. Fondazione Pietro Bembo, Ugo Guanda Editore.

Benz, Ernst. (2016 [1968]). Mística y Romanticismo. Las fuentes místicas del Romanticismo alemán (María Tabuyo y Agustín López, Trad.). Siruela.

Berque, Augustin. (1998). “Le japonais”. En François Mattéi (Ed.), Encyclopédie philosophique universelle, IV: le Discourse philosophique (pp. 240-250). PUF.

Berque, Augustin. (2016). "Thinking the Ambient: On the Possibility of Shizengaku (Naturing Science)". En J. Baird Callicott y James McRae (Eds.), Japanese Environmental Philosophy (pp. 13-28). Oxford University Press.

Cestari, Matteo. (1998). “The Knowing Body: Nishida’s Philosophy of Active Intuition”, The Eastern Buddhist, 31(2), 179-208.

Derrida, Jacques. (1993). Chōra. Galilée.

Diels, Hermann; Kranz, Walther (Eds.). (1959-1960). Die Fragmente der Vorsokratiker. Weidmannsche Verlagsbuchhandlung.

Fichte, Johann Gottlieb. (2000). Die Bestimmung des Menschen. Felix Meiner Verlag.

Heidegger, Martin. (1996). El ser y el tiempo (José Gaos, Trad.). FCE. (Obra original publicada en 1927)

Johnson, David. (2019). Watsuji on Nature. Japanese Philosophy in the Wake of Heidegger. Northwestern University Press. 
Kasulis, Thomas P. (2002). Intimacy or Integrity. Philosophy and Cultural Difference. University of Hawai'i Press.

Kasulis, Thomas P. (2019). La filosofía japonesa en su historia (Raquel Bouso, Trad.). Herder.

Krummel, John W. (2015). Nishida Kitaro's chiasmatic chorology. Place of dialectic, dialectic of place. Indiana University Press.

Maraldo, John C. (2017). "Enaction in Cognitive Science and Nishida's Turn of Intuition into Action”. En Japanase Philosophy in the Making I. Crossing Paths with Nishida (pp. 199-225). Chisokudo.

MiLL, John Stuart. (2003). On Liberty (David Bromwich y George Kateb, Eds.). Yale University Press.

NishidA, Kitarō. (1965a). “Basho”「場所」. En Nishida Kitarō Zenshū 『西田幾多郎全 集』[Obras completas de Nishida Kitarō], vol. 3 (pp. 415-477). Iwanami.

NishidA, Kitarō. (1965b). “El estudio de la religión”「宗教学」 en Nishida Kitarō Zensh $\bar{u}$ 『西田 幾多郎全集』[Obras completas de Nishida Kitarō], vol. 15 (pp 223-304). Iwanami.

NishidA, Kitarō. (1995). Indagación del bien (Alberto Luis Bixio, Trad.). Gedisa.

Nishida, Kitarō. (2016). "La lógica del lugar" (Raquel Bouso, Trad.). En J.W. Heisig, Th. P. Kasulis, J. C. Maraldo, R. Bouso (Eds.), La filosofía japonesa en sus textos (pp. 673-682). Herder.

Platón. (1992). “Teeteto” (Álvaro Vallejo Campos, Trad.). En Diálogos V (pp. 137-317). Gredos.

Platón. (1997). “Timeo” (Francisco Lisi, Trad.). En Diálogos VI (pp. 125-261). Gredos.

SALlis, John. (1999). Chorology. On Beginning in Plato's Timaeus. Indiana University Press.

Tremblay, Jacynthe. (2007). Introduction à la philosophie de Nishida. L'Harmattan.

UedA, Shizuteru. (2004). Zen y filosofía (Raquel Bouso, Ed.). Herder.

UnTERSTEINER, Mario. (2008 [1996]). I Sofisti. Mondadori. 
VAN Berkel, Tazuko A. (2013). “Made to Measure: Protagoras' metron”. En Johannes M. van Ophuijsen, Marlein van Raalte, Peter Sork (Eds.), Protagoras of Abdera. The Man, His Measure (pp. 37-67). Brill.

WAtsujı, Tetsurō. (1960). Climate: a philosophical Study (Geoffrey Bownas, Trad.). Japanese National Commission for UNESCO.

WATsujI, Tetsurō. (1962). “Fūdo: Ningengakuteki kōsatsu”「風土：人間学的考察」 (Clima: Un estudio del ser humano). En Watsuji Tetsurō zenshū 『和辻哲郎全 集』[Obras completas de Watsuji Tetsurō], vol. 8. Iwanami.

WAtsuji, Tetsurō. (1988). Climate and Culture. A Philosophical Study (Geoffrey Bownas, Trad.). Greenwood Press.

WAtsuji, Tetsurō. (2006). Antropología del paisaje (Juan Masiá y Anselmo Mataix, Trad.). Sígueme.

Watsuj, Tetsurō. (2011). Fūdo, le milieu humain (Augustin Berque, Pauline Couteau y Kuroda Akinobu, Trads.). CNRS.

Watsuj, Tetsurō. (2015). Uno studio dell'umano (Lorenzo Marinucci, Trad.). Mimesis. 